\title{
Defibrillation in the dental practice
}

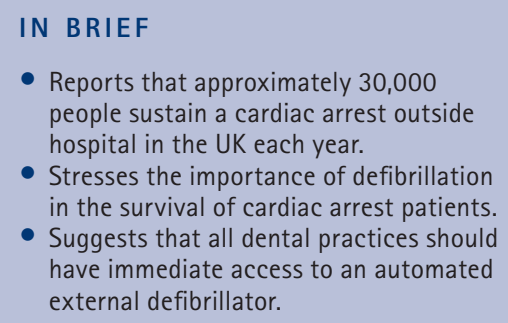

IN BRIEF

Reports that approximately 30,000 people sustain a cardiac arrest outside

Suggests that all dental practices should external defibrillator.

VERIFIABLE CPD PAPER

A large number of cardiac arrests occur outside hospital each year in the UK and as rapid defibrillation plays an integral role in survival rates, it can be seen as all healthcare premises' responsibility to have immediate access to an automated external defibrillator (AED). This article looks at the importance of the AED and the dental practice in emergency resuscitation, and national guidelines.

\section{INTRODUCTION}

About 30,000 people sustain a cardiac arrest outside hospital in the UK each year. ${ }^{1}$ Most of these cardiac arrests are caused by ventricular fibrillation (VF) or pulseless ventricular tachycardia (VT), the definitive treatment of which is rapid defibrillation. ${ }^{2}$ The delay from collapse to defibrillation is the single most important determinant of survival. . $^{3,4}$

Although rare, cardiac arrests do occur in dental practices ${ }^{5}$ and dental staff may need to defibrillate the patient. ${ }^{6}$ The Resuscitation Council (UK) ${ }^{7}$ has therefore recommended that every dental practice should have immediate access to an automated external defibrillator (AED) (Fig. 1).

The aim of this article is to understand the principles of defibrillation in the dental practice.

\section{DEFINITION OF DEFIBRILLATION}

Defibrillation, defined literally as the termination of fibrillation, ${ }^{8}$ involves the passage of an electrical current across the myocardium of sufficient magnitude to depolarise a critical mass of myocardium and enable restoration of coordinated electrical activity. ${ }^{2}$

Resuscitation Officer/Clinical Skills Lead, Mano Hospital, Walsall

Correspondence to: Phil Jevon

Email:phil.jevon@walsallhealthcare.nhs.uk

Accepted 27 June 2012

DOI: 10.1038/sj.bdj.2012.778

${ }^{\circledR}$ British Dental Journal 2012; 212: 233-235

\section{DEFIBRILLATION AND THE CHAIN OF SURVIVAL}

The chain of survival represents the interventions that contribute to a successful outcome after cardiac arrest. A chain is only as strong as its weakest link and the four links of the chain are:

- Early recognition and call for help: to prevent cardiac arrest

- Early CPR: to buy time

- Early defibrillation: to restart the heart

- Post resuscitation care: to restore quality of life.

Defibrillation is arguably the most important link in this chain. Prompt defibrillation can achieve survival rates as high as $75 \%,{ }^{1}$ but the chances of successful defibrillation decline by $10 \%$ each minute it is delayed. ${ }^{9,10}$ Although national standards stipulate that an ambulance reaches $75 \%$ of cardiac arrests within eight minutes, ${ }^{11}$ this usually be too late unless both CPR and defibrillation have been performed. ${ }^{12}$

\section{TYPES OF AEDS}

An AED is a sophisticated, reliable, safe, computerised device that can deliver defibrillatory shocks when appropriate to a victim in cardiac arrest. ${ }^{3}$ Simple to use, it provides voice and/or visual prompts to guide the operator in its use. ${ }^{4}$

AEDs can be classified as semi-automated or automated: ${ }^{4}$

- Semi-automated AED: analyses the

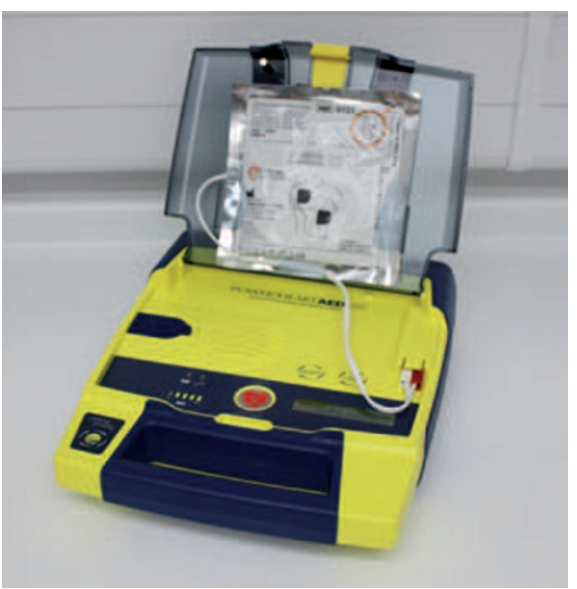

Fig. 1 Automated external defibrillator (AED)

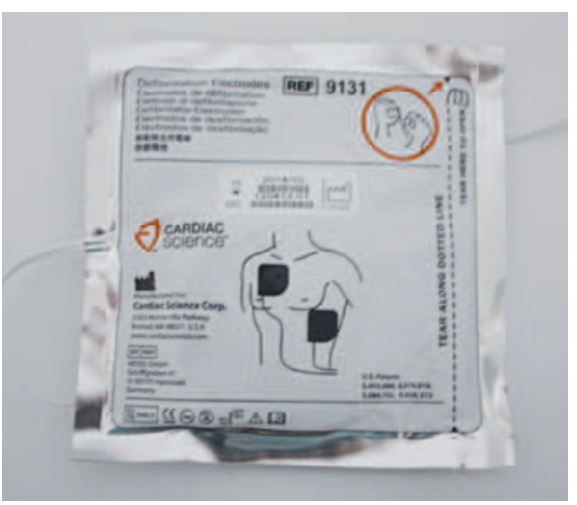

Fig. 2 AED pads

ECG, advises that a shock is required, charges up automatically and prompts the operator to press the 'shock button' to defibrillate the patient

- Automated AED: analyses the ECG, charges up and delivers the shock automatically (the operator is not required to press a 'shock button'). 
Defibrillator technology is rapidly advancing; AED interaction with the operator through voice prompts is now established and future technology may enable more specific instructions to be provided. ${ }^{2}$

\section{AED PADS}

AED pads (Fig. 2) are designed to adhere firmly to the patient's bare chest, providing an effective interface with the patient's skin, allowing the AED to analyse the ECG. The pads have a picture on them advising the user of their correct placement and it does not matter if their positions are reversed. ${ }^{3}$

If the patient has an implanted medical device, for example a permanent pacemaker or implantable cardioverter defibrillator (ICD), under the right clavicle, an alternative AED pad position is advised, such as the anterior-posterior position. ${ }^{4}$

\section{SAFETY PRECAUTIONS RELATING TO AED USE}

There are two key safety precautions relating to AED use:

- Ensuring no one is touching the patient or anything in contact with the patient for example, dental chair, when the shock is delivered

- Temporarily removing face mask/ supplemental oxygen at least one metre away from the patient.

\section{SUGGESTED PROCEDURE FOR USING AN AED}

The following procedure for using an AED is based on the Resuscitation Council (UK) algorithm for automated external defibrillation.

1. Confirm cardiac arrest, start CPR (chest compressions first) at a ratio of 30 compressions: 2 ventilations while a colleague calls 999 for an ambulance and another fetches the AED (and resuscitation equipment)

2. Once the AED arrives, place it near to the patient (for example, on his lap if he is in the dental chair), switch it on and follow the audio and/or visual instructions; advise colleagues to continue with CPR

3. Expose the patient's chest; quickly dry the skin if necessary and apply self adhesive AED pads to the patient's bare chest as instructed (if there is excessive chest hair, quickly remove using a razor, though this should not delay defibrillation if one is not immediately available). ${ }^{3}$ Some AED models require the operator to connect the leads attached to the AED pads to the AED. Advise colleagues to continue CPR while the pads are being applied

4. Once the AED starts to analyse the patient's ECG, stop CPR and follow the prompts; ensure colleagues are not touching the patient during the analysis process

5. If shock is advised shout 'stand clear' and perform a quick visual check to ensure all staff are clear

6. Press shock button as indicated (if AED is automated, it will deliver the shock automatically)

7. Continue CPR 30:2, as guided by the voice/visual prompts.

8. Continue to follow the AED prompts until the paramedics arrive and take over or if the patient displays signs of life for example, coughing, eyes opening, talking or moving purposefully as well as staring to breathe normally. ${ }^{1,4}$

\section{USE OF AEDS IN CHILDREN}

The Resuscitation Council (UK) ${ }^{3}$ has clarified guidance for the use of AEDs in children:

- Persons >8 years of age: standard adult-sized AED pads should be used

- Children aged 1 to 8 years: paediatric AED pads that attenuate

the current delivered during defibrillation should ideally be used (if not available, standard adult-sized AED pads can be used)

- Infants and children < 1 year of age: AED use is not recommended; the use of an AED may be considered if it is the only defibrillator available (ideally with paediatric pads that attenuate the current - see above).

\section{STORAGE AND MAINTENANCE OF AEDS}

The AED should not be locked away, but instead should be easily accessible. The national AED sign could be displayed to highlight the location of the AED.

The AED should be checked and maintained following the manufacturer's recommendations. This typically involves regular checks ensuring the AED is 'rescue ready' and that the AED pads are not outof-date, as well as periodically switching it on. ${ }^{4}$ AEDs generally undertake self checks on a regular basis and will alert (for example, make a bleeping noise) staff if there is a problem such as the battery getting low.

\section{AED TRAINING}

Training and re-training in the use of an AED is variable. ${ }^{13}$ A survey undertake by Day et al. ${ }^{13}$ showed that in over half of health centres that have an AED, clinical staff reported that they had not received AED update training. Poor retention of resuscitation skills following training for both nursing and medical staff is well documented $;{ }^{14}$ regular updates are required to maintain competence and proficiency. ${ }^{15}$

The new Resuscitation Council (UK) ${ }^{3}$ guidelines for resuscitation contain two key statements regarding training in the use of an AED:

- As AEDs can be safely and effectively used without previous training, their use should not be restricted to those trained in their use. However, training is still advocated because user awareness will hopefully instill confidence, ensure correct positioning of electrodes and speed up delivery of the first shock

- Validated short video/computer selfinstruction courses, which involve only minimal or even no training by an instructor as well as hands-on practice, may be considered an appropriate alternative to instructorled CPR/AED courses.

\section{AEDS IN DENTAL PRACTICES - A LEGAL REQUIREMENT?}

It is recommended that every dental practice should have immediate access to an $\mathrm{AED}^{16}$ (Fig. 1). However, under English or Welsh Law, there is no statutory legal requirement to provide a defibrillator. ${ }^{13}$

However, there are implications under common law if adequate safeguards are not provided to protect the public within a facility. ${ }^{13}$ In addition, the GDC's Standards for dental professionals ${ }^{17}$ stresses the importance of maintaining professional knowledge and competence - it could be argued that having an AED immediately available and being competent at using it is in line with the GDC's requirements.

While the question of whether or not to 
have an AED in the dental practice continues to be debated, ${ }^{8,18,19}$ if a patient has a cardiac arrest and dies in a dental practice that does not have an AED, questions will be certainly be asked.

A downloadable poster for your practice entitled 'Resuscitation in the dental practice' is available as supplementary information in the online version of this paper on the BDJ website www.nature.com/bdj.

1. Nolan J P, Soar J, Zideman D A et al. European Resuscitation Council guidelines for resuscitation 2010 section 1. Executive summary. Resuscitation 2010; 81: 1219-1276.

2. Deakin C D, Nolan J P, Sunde K, Koster R W. European Resuscitation Council guidelines for resuscitation 2010 section 3 . Electrical therapies: automated external defibrillators, defibrillation, cardioversion and pacing. Resuscitation 2010; 81: 1293-1304.

3. Resuscitation Council (UK). Resuscitation guidelines 2010. London: NICE, 2010. Online guidelines available at http://www.resus.org.uk/pages/mediMain. htm (accessed August 2012).

4. Jevon P. Defibrillation 1: using an AED outside hospital. Nurs Times 2011; 107: 14-16.

5. Müller M P, Hänsel M, Stehr S N, Weber S, Koch T. A state-wide survey of medical emergency management in dental practices: incidence of emergencies and training experience. Emerg Med J 2008;

25: 296-300

6. Chapman P J, Penkeyman H W. Successful defibrillation of a dental patient in cardiac arrest Aust Dent J 2002; 47: 176-177.

7. Resuscitation Council (UK). Medical emergencies and resuscitation: standards for clinical practice and training for dental practitioners and dental care professionals in general dental practice. London: Resuscitation Council (UK), 2012

8. Leitch J, Schmulian C, Scott A. Automatic external defibrillators - time for a change? Br Dent J 2005; 198: 209-210.

9. Valenzuela T D, Roe D J, Cretin S, Spaite D W, Larsen M P. Estimating effectiveness of cardiac arrest interventions: a logistic regression survival model. Circulation 1997: 96: 3308-3313.

10. Waalewijn R A, de Vos R, Tijssen J G, Koster R W. Survival models for out-of-hospital cardiopulmonary resuscitation from the perspectives of the bystander, the first responder and the paramedic.
Resuscitation 2001; 51: 113-122.

11. Lambert J. The use of automated external defibrillators. Practice Nursing 2006; 17: 1-4.

12. Virdi G, Watson L, Donohoe R. London Ambulance Service cardiac arrest annual report 2008/09. London: London Ambulance Service NHS Trust, 2009.

13. Day A, Oldroyd C, Godfrey S, Quinn T. Availability of cardiac equipment in general practice premises in a cardiac network: a survey. $\mathrm{Br} J \mathrm{Cardio} / 2008$; 15: 141-144.

14. Hamilton R. Nurses' knowledge and skill retention following cardiopulmonary resuscitation training: a review of the literature. J Adv Nurs 2005; 51: 288-297.

15. Woollard M, Whitfield R, Smith A et al. Skill acquisition and retention in automated external defibrillator (AED) use and CPR by lay responders: prospective study. Resuscitation 2004; 60: 17-28.

16. Resuscitation Council (UK). The legal status of those who attempt resuscitation. London: Resuscitation Council (UK), 2010. Online article available at https://www.resus.org.uk/pages/legal.htm (accessed August 2012).

17. General Dental Council. Standards for dental professionals. London: GDC, 2009.

18. Manek P. AED value. Br Dent J 2011; 210: 501

19. Moore R S. Misses the point. Br Dent J 2011; 211: 103. 